\title{
The stellar population and complex structure of the bright-rimmed cloud IC $1396 \mathrm{~N}^{\star}$
}

\author{
M. T. Beltrán ${ }^{1}$, F. Massi², R. López ${ }^{3}$, J. M. Girart ${ }^{4}$, and R. Estalella ${ }^{3}$ \\ 1 Universitat de Barcelona, Departament d'Astronomia i Meteorologia, Unitat Associada a CSIC, Martí i Franquès 1, \\ 08028 Barcelona, Catalunya, Spain \\ e-mail: mbeltran@am.ub.es \\ 2 INAF - Osservatorio Astrofisico di Arcetri, Largo E. Fermi 5, 50125 Firenze, Italy \\ 3 Departament d'Astronomia i Meteorologia, Universitat de Barcelona, Martí i Franquès 1, 08028 Barcelona, Catalunya, Spain \\ ${ }^{4}$ Institut de Ciències de l'Espai (CSIC-IEEC), Campus UAB, Facultat de Ciències, Torre C-5, 08193 Bellaterra, Catalunya, Spain
}

Received 17 December 2008 / Accepted 23 February 2009

\begin{abstract}
Context. IC $1396 \mathrm{~N}$ is a bright-rimmed cloud associated with an intermediate-mass star-forming region, where a number of HerbigHaro objects, $\mathrm{H}_{2}$ jet-like features, $\mathrm{CO}$ molecular outflows, and millimeter compact sources have been observed.

Aims. We study the complex structure of the IC $1396 \mathrm{~N}$ core and the molecular outflows detected in the region in detail and reveal the presence of additional YSOs inside this globule.

Methods. We carried out a deep survey of the IC $1396 \mathrm{~N}$ region in the $J, H, K^{\prime}$ broadband filters and deep high-angular resolution observations in the $H_{2}$ narrowband filter with NICS at the TNG telescope. The completeness limits in the 2MASS standard are $K_{\mathrm{s}} \sim 17.5, H \sim 18.5$, and $J \sim 19.5$.

Results. A total of 736 sources have been detected in all three bands within the area where the $J H K^{\prime}$ images overlap. There are 128 sources detected only in $H K^{\prime}, 67$ detected only in $K^{\prime}$, and 79 detected only in $J H$. We found only a few objects exhibiting a near-infrared excess and no clear signs of clustering of sources towards the southern rim. In the case of triggered star formation in the southern rim of the globule, this could be very recent, because it is not shown through Near-Infrared imaging alone. The $\mathrm{H}_{2}$ emission is complex and knotty and shows a large number of molecular hydrogen features spread over the region, testifying to recent starformation activity throughout the whole globule. This emission is resolved into several chains or groups of knots that sometimes show a jet-like morphology. The shocked cloudlet model scenario previously proposed to explain the V-shaped morphology of the $\mathrm{CO}$ molecular outflow powered by the intermediate-mass YSO BIMA 2 seems to be confirmed by the presence of $\mathrm{H}_{2}$ emission at the position of the deflecting western clump. New possible flows have been discovered in the globule, and some of them could be very long. In particular, the YSO BIMA 3 could be powering an old and poorly collimated outflow.
\end{abstract}

Key words. ISM: individual objects: IC 1396N, IRAS 21391+5802 - ISM: jets and outflows - ISM: lines and bands - infrared: ISM - stars: formation

\section{Introduction}

Bright-rimmed clouds (BRCs) found in HII regions are potential sites of triggered star formation due to compression by ionization/shock fronts. Many of them are associated with IRAS point sources with cold color indices (low dust temperature), which are most probably young stellar objects (YSOs) or protostars. Such clouds are of deep interest from the point of view of ongoing star formation. They frequently contain a small cluster of near-infrared (NIR) stars that is elongated toward the bright-rim tip or the ionizing star(s) of the HII region with the IRAS sources situated near the other end. There is a tendency for bluer (i.e., older) stars to be located closer to the ionizing star(s), and for redder (i.e., younger) stars to be closer to the IRAS sources. This asymmetric distribution of the cluster members strongly suggests small-scale sequential star formation or propagation of star formation from the side of the ionizing star(s) to the IRAS position in a few times $10^{5} \mathrm{yr}$, as a result of the advance of the shock

* Tables 1-5 are only available in electronic form at the CDS via anonymous ftp to cdsarc.u-strasbg.fr $(130.79 .128 .5)$ or via http://cdsweb.u-strasbg.fr/cgi-bin/qcat?]/A+A/504/97 caused by the UV radiation from the ionizing star(s) (Sugitani et al. 1995). Thus, BRCs represent one of the best laboratories for studying the star-formation process at different evolutionary stages.

A good example of BRC with ongoing star-formation activity is IC 1396N (BRC38; Sugitani et al. 1991), located in the Cep OB2 association at a distance of 750 pc (Matthews 1979), and exposed to UV radiation from the O6.5 star HD 206267. The region is associated with IRAS $21391+5802$, a very young intermediate-mass object with a luminosity of $235 L_{\odot}$ (Saraceno et al. 1996), which is powering an extended CO bipolar outflow (Sugitani et al. 1989). Beltrán et al. (2002) have resolved the millimeter emission towards IRAS 21391+5802 into an intermediate-mass source named BIMA 2 surrounded by two less massive and smaller objects, BIMA 1 and BIMA 3. Recent higher angular resolution millimeter interferometric observations have revealed that the intermediate-mass protostar BIMA 2 in fact consists of multiple compact sources (Neri et al. 2007). The gas emission surrounding IRAS $21391+5802$ traces different molecular outflows (Codella et al. 2001; Beltrán et al. 2002, 2004), some of them possibly powered by still undetected 
YSOs (Beltrán et al. 2004). Beltrán et al. (2002) have conducted a detailed study of the bipolar outflow associated with the intermediate-mass protostar BIMA 2, and shown that its complex morphology and kinematics are possibly the result of the interaction between the outflow and the dense cores surrounding the protostar. NIR images of the region have also revealed a number of small scale molecular hydrogen and Herbig-Haro (HH) flows (Nisini et al. 2001; Sugitani et al. 2002a; Reipurth et al. 2003; Caratti o Garatti et al. 2006). This evidence of ongoing star-formation activity at the head of the cometary globule, together with the relatively proximity of the region, make IC $1396 \mathrm{~N}$ one of the best candidates for studying potential sequential star formation.

To do a complete and uniform census of the young stellar population in the globule and reveal the presence of additional young sources inside the globule, deep NIR observations at $J, H$, and $K^{\prime}$ have been carried out. In addition, deep high angular resolution observations in the $\mathrm{S}(1) v=1-0$ line of $\mathrm{H}_{2}$ at $2.12 \mu \mathrm{m}$ have also been performed to investigate the complex structure of this globule, and the morphology of the shocked gas that traces the interaction between the outflows in the region and the dense clumps surrounding the YSOs. The results of this NIR study are presented here.

\section{Observations and data reduction}

The images were taken with NICS (Baffa et al. 2001) at the 3.58-m Telescopio Nazionale Galileo (TNG) telescope (ORM, La Palma, Spain) through the standard $J, H, K^{\prime}$ broadband filters and the $\mathrm{H}_{2}$ narrowband filter centered at $2.12 \mu \mathrm{m}$, during the nights between 16-17 July 2005 . The plate scale is $0.25^{\prime \prime} /$ pixel, yielding a field of view of $\sim 4.2 \times 4.2 \mathrm{arcmin}^{2}$. Both in $K^{\prime}$ and in $\mathrm{H}_{2}$, two positions roughly $100^{\prime \prime}$ apart (east-west) were imaged, as to have an overlapping field, $\sim 150^{\prime \prime}$ wide (in RA), enclosing the globule. In $H$, the two imaged positions are separated by $\sim 50^{\prime \prime}$ east-west, so the overlapping field is $\sim 200^{\prime \prime}$ wide. Due to shortage of time, only one field could be imaged in $J$, centered on the globule. The seeing was $\sim 0.8^{\prime \prime}$ in $K^{\prime}, H$ and $\mathrm{H}_{2}$, and $\sim 0.9^{\prime \prime}$ in $J$. In $K^{\prime}$ and $H$, groups of five on-source integrations with a dithering of up to $10^{\prime \prime}$ in RA and Dec were interspersed between groups of five off-source integrations. The off-source fields are located $\sim 6^{\prime}$ from the target and were chosen through examination of 2MASS images. The dithering of the off-source frames is up to $20^{\prime \prime}$ in RA and Dec. In $J$, groups of two on-source images were interspersed between groups of two off-source images. Ditherings and off-source fields are the same as above. Finally, in $\mathrm{H}_{2}$, pairs of one on-source and one off-source images, with the same ditherings and off-source fields as above, were taken. Each frame was integrated 5 or $10 \mathrm{~s}$ in $K^{\prime}$, depending on the background level; the total integration time is $600 \mathrm{~s}$ for each of the two positions. At $H$, each individual integration is $20 \mathrm{~s}$ and the total integration time is $600 \mathrm{~s}$ for each of the two positions, as well. At $J$, each individual integration is $100 \mathrm{~s}$ and the total integration time is $600 \mathrm{~s}$. In $\mathrm{H}_{2}$, the individual integration times are 100 or $150 \mathrm{~s}$, depending on the background level, and the total integration time is $2700 \mathrm{~s}$ for each of the two positions.

Each frame was first corrected for cross-talk using the routine provided on the TNG web page (http://www. tng.iac. es). Data were then reduced in the standard way by using IRAF ${ }^{1}$

1 IRAF is distributed by the National Optical Astronomy Observatories, which are operated by the Association of Universities for Research in Astronomy, Inc., under cooperative agreement with the National Science Foundation. routines. Flat-field frames were acquired at sunset. Differential flat-field images were constructed for $K^{\prime}$ and $\mathrm{H}_{2}$, whereas all available frames with roughly the same mean level of counts were averaged together for $H$ and $J$. All on-source and offsource frames were then flat-field corrected. Sky frames were constructed by median-averaging the six off-source frames closest to each on-source frame (generally, three preceding and three following), after removal of the imaged stars. The sky frames were then subtracted from the corresponding on-source frames. At $K^{\prime}$ and $\mathrm{H}_{2}$, the sky-subtracted images were multiplied by a factor when obtained with different individual exposure times, as to "convert" the counts of all frames in the same band to a same exposure time. After bad-pixel correction, all images in a same band were registered and combined by using a median filter. The composite three-color $J H K^{\prime}$ image of the area where the $J H K^{\prime}$ frames overlap is shown in Fig. 1.

Photometry on all mosaiced images was performed by using DAOPHOT (in IRAF). The detected stars were retrieved by running DAOFIND and, subsequently, by a visual check to discard fake detections and add undetected faint sources. Aperture photometry was carried out through PHOT, by adopting an aperture $\sim 1 F W H M$ in radius and an annulus $\sim 2 F W H M$ wide with an inner radius $\sim 2 F W H M$. The weather was barely photometric, so the calibration was performed by cross-correlating the sources found in the $J H K^{\prime}$ bands and the 2MASS point source cata$\log$. A linear relation in the $J-H$ or $H-K^{\prime}$ instrumental colors was fitted to these pairs of instrumental magnitude and 2MASS magnitude and the corresponding instrumental color. Hence, the given magnitudes are in the 2MASS system $\left(J H K_{\mathrm{s}}\right)$. The color coefficient is always less than 0.06 in each band. As a check of consistency, we compared our $K_{\mathrm{s}}$ photometry and that of Nisini et al. (2001) for the isolated sources out of those listed by those authors. Our $K_{\mathrm{s}}$ values are on average $0.34 \pm 0.28$ mag dimmer than those by Nisini et al. (2001). This is very likely due to the much worse seeing $\left(\sim 2-3^{\prime \prime}\right)$ and the much coarser sampling $\left(\sim 1^{\prime \prime}\right)$ of the PSF in the data reported by Nisini et al. (2001), given the highly variable background level in the region.

Additional photometry of the detected $\mathrm{H}_{2}$ features was performed from the narrowband $\mathrm{H}_{2}$ image. The $\mathrm{H}_{2}$ filter is centered on the $2.12 \mu \mathrm{m}$ line of molecular hydrogen. Continuum emission falls within the bandpass, as well as line emission. Through photometry in $\mathrm{H}_{2}$ and $K^{\prime}$, we estimated the fraction of stellar continuum affecting the total counts in the $\mathrm{H}_{2}$ frame. First, based on the characteristics of the two filters, two scale factors can be derived by which we multiplied the $\mathrm{H}_{2}$ and $K^{\prime}$ images, and then we subtracted the latter from the former. The resulting subtracted image contains only the line emission falling within the $\mathrm{H}_{2}$ filter. The calibration was performed by carrying out stellar photometry on the $\mathrm{H}_{2}$ original image, the same way as for $J H K^{\prime}$, but also including an aperture correction. The retrieved stars were crosscorrelated with those found in the $H K^{\prime}$ bands and their correct flux was derived by interpolation with the corresponding ones at $H$ and $K_{\mathrm{s}}$ in the 2MASS system. Through a fit, we determined the conversion factor from counts to flux. The detection limit (at a $3 \sigma$ level) is $\sim 10^{-15} \mathrm{erg} \mathrm{cm}^{-2} \mathrm{~s}^{-1} \operatorname{arcsec}^{-2}$. The procedure outlined above yielded an image where all stellar sources were efficiently removed, therefore only containing $\mathrm{H}_{2}$ line emission knots. We classified each local emission peak as a knot and defined a polygon around each knot such as to include emission down to a $\sim 3 \sigma$ limit. Polygon borders for close-by knots were chosen by eye, based on morphological criteria. Photometry was carried out by using POLYPHOT in IRAF.

The astrometric calibration was performed by deriving the positions of 14 relatively bright, isolated stars spread over all the 


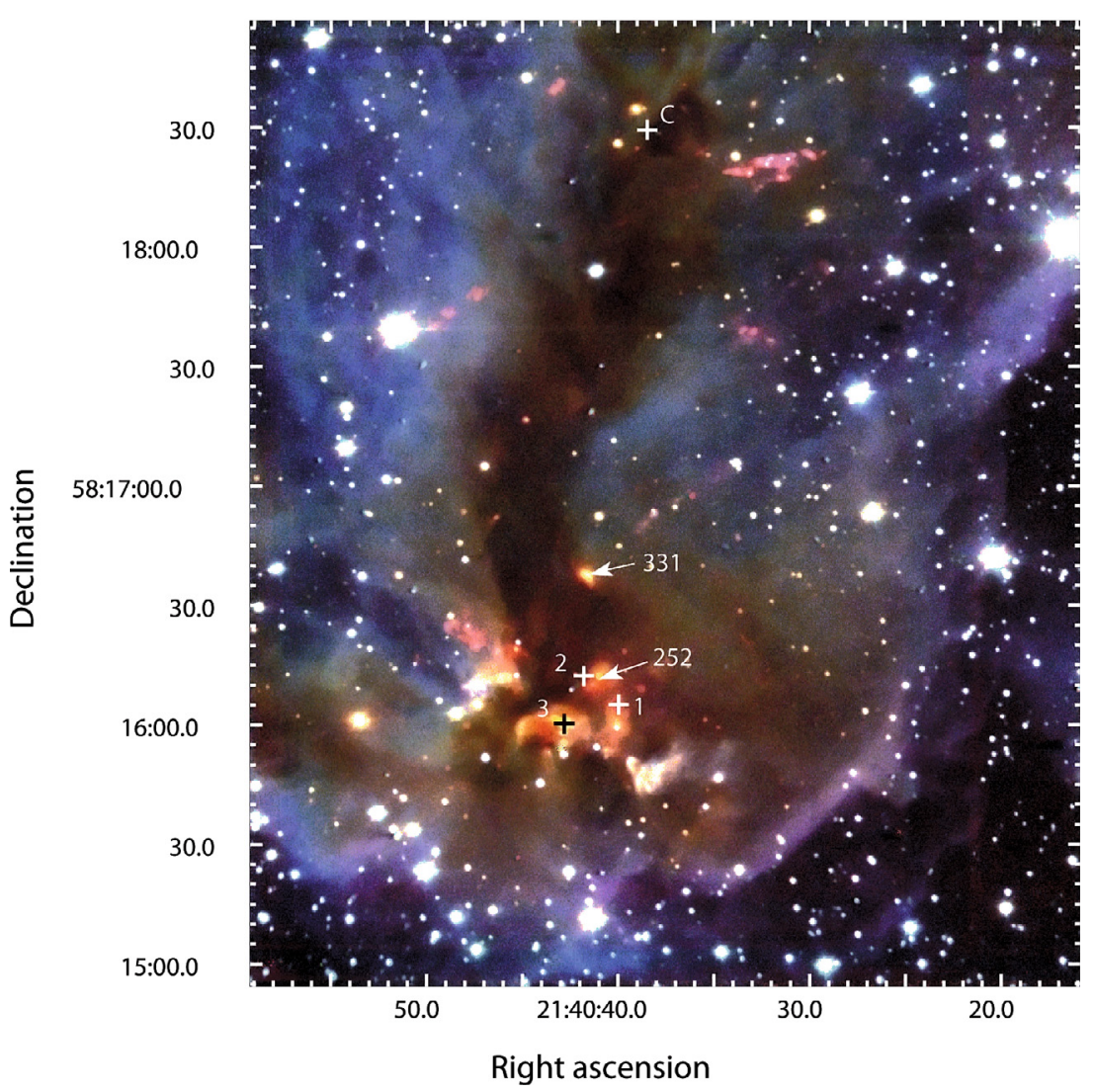

Fig. 1. Three-color composite image of IC $1396 \mathrm{~N}\left(J\right.$, blue, $H$, green, $K^{\prime}$, red) taken with NICS at TNG. The black and white crosses show the positions of the $3.1 \mathrm{~mm}$ sources, BIMA 1, 2, and 3 from Beltrán et al. (2002), while the white cross at the top shows the position of the $1.3 \mathrm{~mm}$ continuum source $\mathrm{C}$ detected by Codella et al. (2001). Also labeled are two Class I sources discussed in the text.

$K^{\prime}$ frame, and correlating them with their 2MASS coordinates. By a fit (using STSDAS routines in IRAF) we obtained the transformation between frame and equatorial coordinates, allowing an accuracy of $\sim 1^{\prime \prime}$.

\section{Results and discussion}

\subsection{The stellar population}

Within the area where the $J H K^{\prime}$ images overlap (see Fig. 1), we found 736 sources detected in all three bands, 128 sources detected only in $H K^{\prime}, 67$ sources only with a $K^{\prime}$ detection, and 79 sources detected only in $J H$. The sources with $H K^{\prime}$ or $K^{\prime}$ detections only are primarily located towards the globule (see, e.g. Fig. 4), as expected for heavily extincted stars. Conversely, the sources with $J H$ detections tend to be located outside the globule, indicating that these are just faint stars.

We obtained histograms of the number of sources as a function of magnitude by binning the number of sources detected in all three bands in magnitude intervals. Then, as a completeness limit in each band we adopted the magnitude where the corresponding histogram peaks: $K_{\mathrm{s}} \sim 17.5, H \sim 18.5$, and $J \sim 19.5$. When also adding the sources with detections in only two or one bands, the peak does not shift in any of the histograms but $K_{\mathrm{s}}$, where it appears to move towards $K_{\mathrm{s}} \sim 18$. The derived completeness limits are roughly $1.5 \mathrm{mag}$ below our detection limits (at a $3 \sigma$ level). An estimate of the minimum stellar mass detectable all over the globule can be obtained by using pre-main sequence (PMS) evolutionary tracks. However, one has to assume an age and a maximum extinction for the stellar population. As for the age, Lada \& Lada (2003) noted that the embedded phase of star cluster evolution lasts 2-3 Myr, and clusters older than $5 \mathrm{Myr}$ are rarely associated with molecular gas. This is in accord with the age of the open star cluster Trumpler 37 surrounding the globule $\left(\sim 3 \times 10^{6} \mathrm{yr}\right.$; Getman et al. 2007). The age of the star exciting the PDR around the globule may also give a hint of the age of the stellar population, since this star either triggered star formation in the core or began inhibiting it by starting core destruction. If HD 206267 is an O6.5 V star (Walborn \& Panek 1984), then its lifetime in the main sequence is $\sim 6 \times 10^{6} \mathrm{yr}$ (e.g. Vanbeveren et al. 1998), which roughly agrees with the times given above. On the other hand, the globule shows the clear signature of much younger stars and protostars, and the dynamical timescales estimated from the jets (see Sect. 3.3) are even shorter, $\sim 10^{3} \mathrm{yr}$. We can therefore assume an age of $10^{6} \mathrm{yr}$ as a sort of upper limit, since younger low-mass PMS stars are brighter and, then, more easily detectable (lowering the mass detection limit). As for the maximum extinction, Getman et al. (2007) quote a few authors to conclude that the absorption through the core is $A_{V} \sim 9-10 \mathrm{mag}$. Figures 2 and 3 clearly show that this value is probably too low and most of the detected stars exhibit $A_{V} \leq 20 \mathrm{mag}$. Nevertheless, there are a few sources with $A_{V}$ up to $\sim 30 \mathrm{mag}$. More extincted sources could not be represented in the diagrams because just too faint, thus biasing any estimates based on the plots. In fact, towards BIMA 2, we can derive an extinction much greater than 100 mag from the continuum millimeter data; however, this is clearly a less evolved, very young region amounting to a small fraction of the globule. We can probably assume that for most of the globule the extinction does not exceed a canonical $A_{V} \sim 30-40 \mathrm{mag}$. 


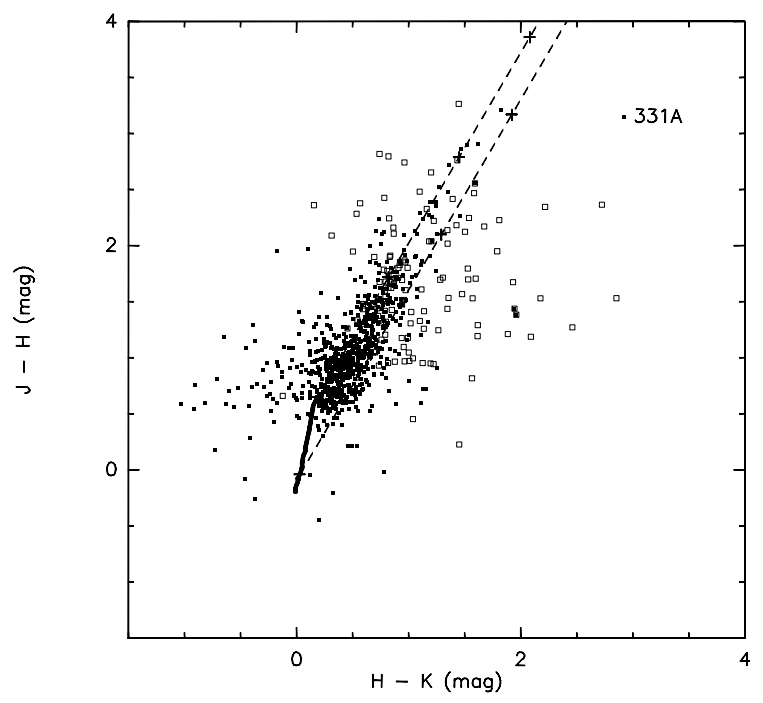

Fig. 2. Color-color diagram of the NIR sources found within the area where $J H K^{\prime}$ images overlap. Full squares are sources with detection in all bands, empty squares are sources with detection in $H K_{\mathrm{s}}$ only (hence, the shown $J-H$ is a lower limit), the solid line marks the main sequence (in the 2MASS system), the dashed lines follow the reddening law by Rieke \& Lebofsky (1985) with crosses at intervals of $A_{\mathrm{v}}=10 \mathrm{mag}$.

We adopted the PMS evolutionary tracks by Palla \& Stahler (1999), along with the reddening law by Rieke \& Lebofsky (1985). Hence, assuming an age of $10^{6} \mathrm{yr}$, PMS stars of $\sim 0.1 M_{\odot}$ are within the completeness limit at $K_{\mathrm{s}}$ for $A_{\mathrm{V}}=30 \mathrm{mag}$ and within the detection limit at $K_{\mathrm{s}}$ for $A_{\mathrm{V}}=40 \mathrm{mag}$. The same for PMS stars of $\sim 0.4 M_{\odot}$ at $H$, whereas PMS stars of $\sim 0.8 M_{\odot}$ are within the detection limit at $J$ for $A_{\mathrm{V}}=30 \mathrm{mag}$, and PMS stars of $\sim 2 M_{\odot}$ are within the completeness limit at $J$ for $A_{V}=30 \mathrm{mag}$; however, these magnitudes refer to "naked" stars, i.e., stars without a circumstellar disk.

The color-color diagram (CCD; $J-H$ vs. $H-K_{\mathrm{s}}$ ) of the NIR sources found within the area where $J H K^{\prime}$ images overlap is shown in Fig. 2. The main sequence locus is also drawn by using the colors of Koornneef (1983) after conversion to the 2MASS system through the relations given by Carpenter (2001). The CCD is consistent with the one shown in Nisini et al. (2001), in that most of the stars fall within the reddening band of the main sequence and almost all those exhibiting an NIR excess lie only slightly below the reddening band. The points spread around the main sequence with larger NIR excesses are mostly faint sources found at the edge of the images, hence affected by large errors. However, our source \# 331 (labeled in figure) actually exhibits a large NIR excess. This source coincides with source \# 8 in Nisini et al. (2001) and HH777 IRS in Reipurth et al. (2003). According to these authors, this source could be binary because it seems to be powering two flows, a major HH flow that expands towards the southwest, labeled HH777 by Reipurth et al. (2003), and a northwestern flow labeled G by Nisini et al. (2001). The source is neatly elongated with respect to the PSF of a single star, with the size of the major axis twice that of the minor axis, which suggests the binarity of the source. The nearby stars do not show any such elongation, so we discarded any possible focus effect. The elongation of source \# 331 is very clear in the $H$ filter, in which it has been possible to deconvolve the emission into two stars \# 331A and \# 331B by PSF-fit photometry with DAOPHOT in IRAF. In the $K^{\prime}$ filter the elongation is also evident, but the PSF-fit photometry appears to be less precise. Nevertheless, we cannot rule out the possibility that \# 331B may

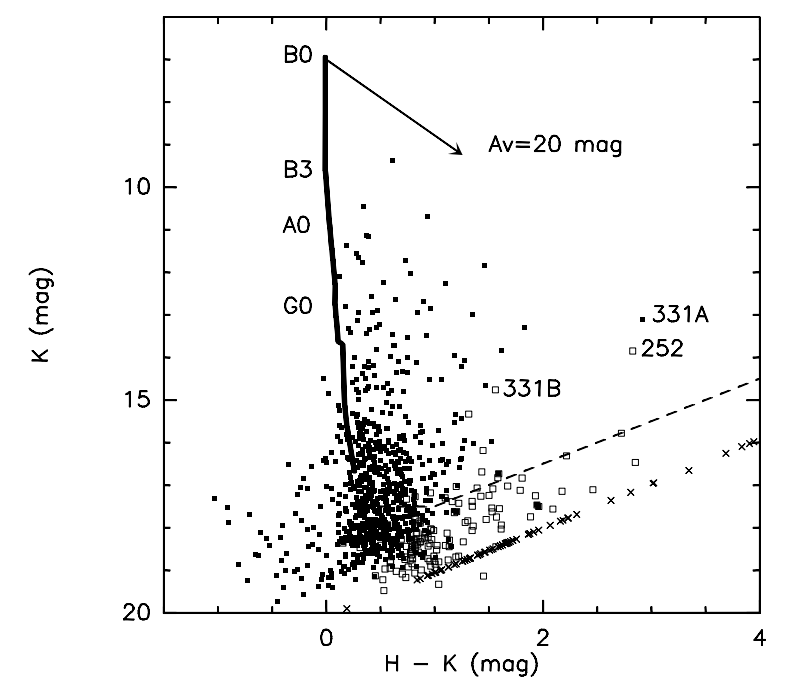

Fig. 3. Color-magnitude diagram of the NIR sources found towards the area where $J H K^{\prime}$ images overlap. Full squares are sources with detections in all bands, empty squares are sources only with detections in $H K_{\mathrm{S}}$ and crosses are sources detected only at $K_{\mathrm{s}}$ (hence, the shown $H-K_{\mathrm{s}}$ is a lower limit). The solid line marks the zero-age main sequence (from Allen (1976) and Koornneef (1983) after conversion to the 2MASS system) at a distance of $750 \mathrm{pc}$, the dashed lines indicate the completeness limit and an arrow is drawn showing a reddening $A_{\mathrm{V}}=20$ mag according to the reddening law by Rieke \& Lebofsky (1985). A few spectral types on the ZAMS are labeled.

just be radiation from \# 331A scattered by dust through a cavity. This scenario would be consistent with the fact that the elongation of source \# 331 roughly coincides with the direction of the southwestern HH777 flow (see Fig. 2 of Reipurth et al. 2003). However, as seen in Figs. 1 and 6, the other $\mathrm{H}_{2}$ flow detected nearby, the northwestern flow labeled $\mathrm{G}$, also points right back towards source \# 331, which suggests that its powering source is also located at that position. Based on the fact that there are two outflows associated with this position, we therefore favor the scenario of binarity to explain the elongation of source \# 331. The $H K_{\mathrm{s}}$ magnitude of \# 331A is within 0.4 mag of those listed in the 2MASS catalog but, whereas its colors are consistent with those given by Nisini et al. (2001), the $K_{\mathrm{s}}$ value we derive is almost 1 mag greater than the one measured by Nisini et al. (2001). This is still consistent with the difference found between the two photometries (see Sect. 2), also given that the source has been resolved into two close-by companions that appear to be embedded in a small diffuse nebulosity that probably could not be resolved by Nisini et al. (2001). However, a degree of intrinsic variability cannot be excluded either.

The color-magnitude diagram $\left(\mathrm{CMD} ; H-K_{\mathrm{s}}\right.$ vs. $\left.K_{\mathrm{s}}\right)$ is shown in Fig. 3 for the NIR sources in the same area as above. As seen in this diagram, an upper limit for the spectral type of the ZAMS stars in the cloud would be B1-B0, which corresponds to a stellar mass of $\sim 17-20 M_{\odot}$ (Vacca et al. 1996). Since such massive stars evolve along the ZAMS from $8-10 M_{\odot}$ on (e.g. Palla \& Stahler 1991) at the end of their accretion phase, this can be considered as a robust upper limit for the mass of the stars associated with IC1396N, irrespective of their age. Most of the points lie within $A_{\mathrm{V}}=20 \mathrm{mag}$ of the zero age main sequence (ZAMS). However, source \# 252 has similar $K_{\mathrm{s}}$ and $H-K_{\mathrm{s}}$ to \# 331A, both objects lying farther from the ZAMS than the remaining stellar population. Source \# 252 is also embedded in a patch of diffuse nebulosity and is located near a cluster of $\mathrm{H}_{2}$-emission blobs already identified by Nisini et al. (2001) as knot A, and the cluster 
of compact radio sources found by Beltrán et al. (2002). These facts suggest that sources \# 252 and \# 331A may be in a similar evolutionary stage, although this cannot be fully proved because \# 252 has not been detected at $J$. They are located towards the center of the globule, 26" apart (see Fig. 1). Source \# 252 lies close to the IRAS uncertainty ellipse, northwest of it. Projecting them back onto the ZAMS in the CMD, along the reddening vector, identify them as stars of spectral type B0 to B3. This has to be considered as a "lower" limit for their actual spectral type (i.e., they are of later spectral type), since \# 331A exhibits an NIR excess and probably \# 252 also has it. Based on the location of \# 331A in the CCD, they might be Class I sources of intermediate mass (e.g. Sugitani et al. 2002b).

Getman et al. (2007) used CHANDRA X-ray observations of IC $1396 \mathrm{~N}$, complemented with Spitzer/IRAC photometry and the available NIR photometry, identifying 25 likely stellar members of the globule. Although all are associated with IRAC MIR sources, 6 of them do not have an NIR counterpart in either the 2MASS catalog or the list of Nisini et al. (2001). We have found three new matches, e.g. sources 70, 76, and 80 (see Table 2 of Getman et al. 2007), corresponding to our sources 224 $\left(K_{\mathrm{s}} \sim 16\right), 223\left(K_{\mathrm{s}} \sim 16\right)$ and $196\left(K_{\mathrm{s}} \sim 17.7\right)$, respectively. None are detected in $J$ or in $H$, confirming their nature of heavily extincted source. The remaining $3 \mathrm{X}$-ray sources without an NIR counterpart lie in the area of the BIMA sources, and their X-ray spectra are heavily absorbed $\left(N_{\mathrm{H}} \gtrsim 10^{23} \mathrm{~cm}^{-1}\right)$. One of them (source 66) has been proposed as an X-ray counterpart to the protostar BIMA 2. However, our sources \# 331A (and $331 \mathrm{~B})$ and 252 do not have any X-ray counterpart in the catalog of Getman et al. (2007). These authors quote a completeness limit (in mass) of $0.4 M_{\odot}$, higher than our completeness limit in $K_{\mathrm{S}}$ but similar to our completeness limit in $H$. We suspect that their completeness limit may be even higher, since the reddening towards the globule may be at least twice greater than adopted by them. It is noteworthy that Getman et al. (2007) find X-ray counterparts of possible Class I sources with high absorption and no NIR counterparts, but fail to detect our sources \# 331A and 252. This would be consistent with \# 331A and 252 being young intermediate-mass (proto-)stars. If \# 331 is actually a double system, then its companion (possibly \# 331B) might be either another young intermediate-mass star or a low-mass protostar (with a mass below the X-ray completeness limit). Moreover, sensitive millimeter interferometric observations did not detect \# 252 (Beltrán et al. 2002; Neri et al. 2007), which rules out the presence of the expected massive enough circumstellar disk. Hence, further data are needed to clarify its nature, and its identification as an intermediate-mass Class I source remains highly speculative.

\subsection{Triggered star formation?}

In the X-ray source population towards IC $1396 \mathrm{~N}$, Getman et al. (2007) find a clear clustering of sources at the southern rim, with an elongated spatial distribution, and an evolutionary gradient (interpreted as an age gradient), oriented towards the exciting star. These authors interpret this geometric and age distribution in terms of triggered star formation by passage of HII region shocks into the molecular globule. We searched for evidence in the NIR of age gradients in the south-north direction or clustering of stars towards the rim, but found none. The number of sources with evidence of NIR excess towards the globule is too low, so any analysis of the stellar population in the NIR alone is bound to remain inconclusive with respect to the identification of age gradients. Regarding the geometric distribution of

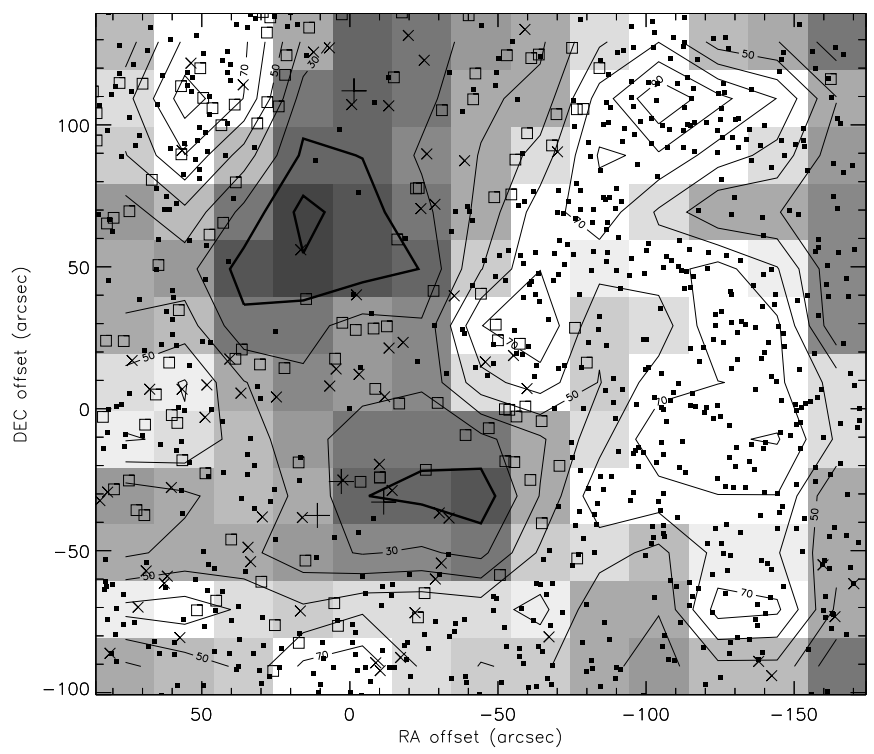

Fig. 4. Stellar-surface density map (in stars $\operatorname{arcmin}^{-2}$ ) of all sources detected in the $K_{\mathrm{s}}$ band up to $K_{\mathrm{s}}=20$. Contours range from 10 to 90 stars $\operatorname{arcmin}^{-2}$ in steps of 10 stars $\operatorname{arcmin}^{-2}$. The contours at 10 and 20 stars $\operatorname{arcmin}^{-2}$ are thicker. Grey scale ranges from -15 stars $\operatorname{arcmin}^{-2}$ (black) to 69 stars $\operatorname{arcmin}^{-2}$ (white). The field shown has been imaged at all bands and the offsets are in arcsec from the location of source \# 331A (HH777 IRS). The positions of sources with detections in all bands are marked by filled squares, those of sources with detections at $H K_{\mathrm{s}}$ only by empty squares, and those of sources with only a $K_{\mathrm{s}}$ detection are marked by small crosses. The three large crosses around $\left(0^{\prime \prime},-30^{\prime \prime}\right)$ are the compact embedded sources BIMA 1, 2, and 3 detected by Beltrán et al. (2002) at millimeter wavelengths, and the one at around $\left(0^{\prime \prime}, 110^{\prime \prime}\right)$ is the $1.3 \mathrm{~mm}$ continuum source $\mathrm{C}$ detected by Codella et al. (2001).

the sources in the NIR, there are no clear signs of clustering towards the rim (even within the area where X-ray sources cluster), as shown by the map of the star surface density (Fig. 4), which was obtained by counting all sources with a detection at least in the $K_{\mathrm{s}}$ band (up to $K_{\mathrm{s}}=20$ ) in squares of $40^{\prime \prime} \times 40^{\prime \prime}$, displaced by $20^{\prime \prime}$ both in RA and in Dec. The number of sources decreases when going from the southern edge of the globule to the northern one; in the CCD, within the extinction band of the main sequence the upper and lower limits of extinction initially increase, then decrease close to the northern edge, as expected. What is more, the $J H K_{\mathrm{s}}$ sources lying below the main sequence reddening band in the $\mathrm{CCD}$ tend to cluster out of the eastern and western edges of the globule, but much less so towards the southern border. As discussed above, our $J H K_{\mathrm{s}}$ colors are biased towards unextincted PMS stars of all masses and heavily extincted intermediate-mass young stars. Instead, $H K_{\mathrm{s}}$ colors only can show heavily extincted PMS stars down to $0.4 M_{\odot}$. These appear to be mostly located towards the globule, but it is still difficult to evidence a significant clustering towards the southern border. A clearer clustering of reddened objects occurs towards the center of the globule. This is also visible as an increase in surface density (Fig. 4) north of sources \# 331 (A and B), mostly due to sources with $H K_{\mathrm{s}}$ or $K_{\mathrm{s}}$ detection alone.

Therefore, only very few or no sources with an NIR excess clusters towards the southern rim, as far as extinction is low and we are sensitive to very low masses. Of course, a number of stars with an NIR excess may concentrate north of the southern edge of the globule, towards the BIMA sources, were extinction is much higher. Then, any triggered YSOs may still be too young to be indicated through NIR imaging alone. This would be 
consistent with the star-surface density map (Fig. 4), which shows two surface-density minima towards the globule, one in the southern part and one in the northern part. The southern one is close to the position of the embedded sources BIMA 1, BIMA 2, and BIMA 3 detected by Beltrán et al. (2002), and the northern one lies along the gas elongation visible in CO, CS (Codella et al. 2001), and $\mathrm{H}^{13} \mathrm{CO}^{+}$(Sugitani et al. 2002a). These two surface density minima outline the more extincted (densest) parts of the globule, not yet visible at NIR wavelengths.

By decreasing the limiting magnitude to $K_{\mathrm{s}}=18$ (i.e., the completeness limit), one obtains a similar surface density map, but the "bridge" of sources crossing the two minima almost disappears, confirming it mostly arises from the detection of faint sources. If this were a real group of young stars associated with the globule, then their birth could hardly be explained as triggered, since they are located north of the group of the youngest protostars (the three compact radio sources found by Beltrán et al. 2002), farther from the ionization front. What is more, in going from south to north, one finds the cluster of Class $0 / \mathrm{I}$ sources observed at millimeter wavelengths by Beltrán et al. (2002) and Neri et al. (2007), then source \# 331A, which is definitely a more evolved object, and then millimeter source $\mathrm{C}$ (Codella et al. 2001), which is a deeply embedded and very young object. Therefore, it is clear from the NIR and millimeter observations that not all the star formation in the globule can be explained in terms of triggering.

The difficulty of proving that any stellar population is associated with the globule by means of NIR photometry alone has already been noted by Getman et al. (2007). As discussed, X-ray observations proved much more efficient in selecting this population, although it clearly failed to probe all young stars and protostars. By combining X-ray and Spitzer/IRAC observations, they could indeed find a number of Class I sources towards the globule. This confirms what we inferred from our NIR images. Also, those authors estimate that the total population of T Tauri stars of the globule is $\sim 30$, also consistent with the lack of a significant increase in the NIR source surface density towards the rim. Nevertheless, the lack of sources exhibiting a clear NIR excess close to the rim is unusual for a stellar population of $\sim 10^{6} \mathrm{yr}$ old and strongly suggests that the intense UV radiation may have affected their circumstellar environments, suddenly stopping their growth. This would be confirmed by the low masses inferred for the counterparts of the X-ray sources associated with the globule. Most of them have estimated masses between $0.2-0.5 M_{\odot}$, and they would be even less massive if they were younger than $10^{6} \mathrm{yr}$, as assumed by Getman et al. (2007). We therefore caution against interpreting an evolutionary gradient as an age gradient in an environment like the edge of a bright-rimmed cloud. The eroding ionization front, in fact, may have dispersed the circumstellar environment of protostars downstream of it leaving them as naked stars, without significantly affecting the protostars upstream of it. In this case, an evolutionary gradient may not correspond to a real age gradient. As shown, star formation activity is present throughout the whole core, even in the northern part. Then, at the moment it appears really difficult to prove that star formation in the southern rim has been triggered.

\section{3. $\mathrm{H}_{2}$ emission}

Figure 5 shows the $2.12 \mu \mathrm{m} \mathrm{H}_{2}$ integrated line emission in greyscale overlaid with an IRAC $4.5 \mu \mathrm{m}$ image in color obtained from the Spitzer Center Archive using the Leopard software. The title of the Spitzer program is Star Formation in Bright Rimmed
Clouds, and the principal investigator is Giovanni Fazio. The CO $(J=1 \rightarrow 0)$ emission integrated in the low-velocity outflow interval $[ \pm 3.5, \pm 9.5] \mathrm{km} \mathrm{s}^{-1}$ is also shown in red and blue contours (Beltrán et al. 2002). Figure 6 shows a close-up of the $\mathrm{H}_{2}$ emission line features. As seen in these figures, the $\mathrm{H}_{2}$ emission shows a large number of molecular hydrogen features spread over the region. Many of these emission features have already been found by Nisini et al. (2001), Reipurth et al. (2003), and Caratti o Garatti (2006). We continued and expanded the nomenclature started by these authors when new $\mathrm{H}_{2}$ features were discovered (see Table 5). Most of the $\mathrm{H}_{2}$ features are also visible in the IRAC $4.5 \mu \mathrm{m}$ image (Fig. 5). That the IRAC $4.5 \mu \mathrm{m}$ band is very efficient in detecting Herbig-Haro objects is due to the spectral response function being highest in this band, and to the existence of many vibrational and rotational $\mathrm{H}_{2}$ emission lines between 4-5 $\mu \mathrm{m}$ (see, e.g. Smith \& Rosen 2005). In addition, the $4.5 \mu \mathrm{m}$ band is less affected by polycyclic aromatic hydrocarbons (PAHs), whose emission could hide the shock-excited $\mathrm{H}_{2}$ features of the $\mathrm{HH}$ flows.

The $\mathrm{H}_{2}$ emission is not smooth but complex and knotty, with several condensations embedded in a more diffuse and nebular emission (Fig. 6). The deep and sub-arcsecond resolution $\mathrm{H}_{2}$ observations have allowed us to resolve the emission into several chains of knots that could trace different flows. Particularly interesting are the chains of knots labeled A, B, and C, for which more than 10 individual knots have been mapped. The peak position and photometry of the individuals knots are given in Table 5. We have denoted the individual components of the different $\mathrm{H}_{2}$ emission line features by numbers. These chains or groups of $\mathrm{H}_{2}$ knots sometimes show a jet-like morphology that along with being located in different parts of the globule, not just on the bright rim, suggests that the $\mathrm{H}_{2}$ excitation mostly stems from shocks driven by outflows powered by YSOs (Nisini et al. 2001).

\subsection{1. $\mathrm{H}_{2}$ knots and flows towards the BIMA sources}

Beltrán et al. (2002) observed the region surrounding the intermediate-mass YSO IRAS $21391+5802$ (BIMA 2) in several molecular tracers and continuum at millimeter wavelengths with the BIMA interferometer. These authors resolved the millimeter continuum emission into three sources, BIMA 1, 2, and 3 , and mapped in CO two molecular outflows: a north-south outflow powered by BIMA 1 and an east-west one driven by BIMA 2. The latter outflow shows a very complex morphology and kinematics, with a clear bipolarity at high outflow velocities (with the blueshifted emission towards the west and the redshifted one towards the east), while at low outflow velocities the direction of the outflow gets deflected and the blueshifted and redshifted emission are highly overlapped (see Fig. 7). Beltrán et al. (2002) explain the complexity of this outflow in terms of a shocked cloudlet model scenario, in which the molecular outflow would interact with the dense material surrounding the embedded sources. The outflow is almost on the plane of the sky (Codella et al. 2001 assume an inclination angle of $10^{\circ}-20^{\circ}$ ), which would explain why the red- and blueshifted outflow emission overlaps after the shock.

In this section, we want to study the morphology of the BIMA 2 outflow in more detail and to check the validity of the shocked scenario by comparing the $2.12 \mu \mathrm{m} \mathrm{H}_{2}$ emission with that of the outflow as seen in CO and CS with the BIMA interferometer. The top panel of Fig. 7 shows the $\mathrm{CO}(J=1 \rightarrow 0)$ emission integrated in the low-velocity outflow interval (Beltrán et al. 2002) overlapped on the $2.12 \mu \mathrm{m} \mathrm{H}_{2}$ integrated line emission in grey-scale. The angular resolution of this image is much 


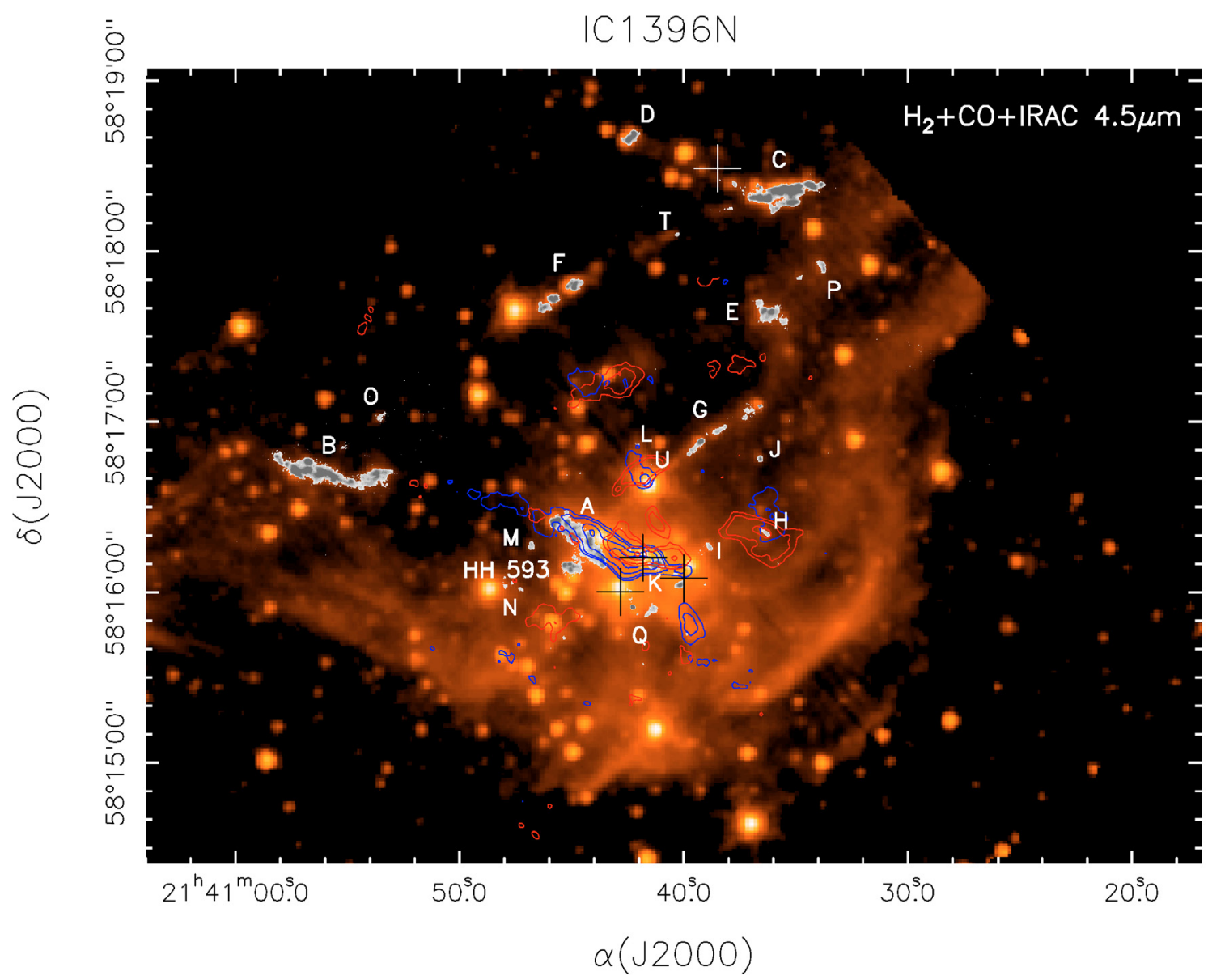

Fig. 5. $\mathrm{H}_{2}(2.12 \mu \mathrm{m})$ image (continuum-subtracted) in greyscale and $\mathrm{CO}(J=1 \rightarrow 0)$ emission in red and blue contours (Beltrán et al. 2002) overlaid with an IRAC $4.5 \mu \mathrm{m}$ image. The $\mathrm{H}_{2}$ emission shows a large number of molecular hydrogen features, many already found by Nisini et al. (2001), Reipurth et al. (2003), and Caratti o Garatti (2006), whose nomenclature we use and expand. The black crosses show the positions of the $3.1 \mathrm{~mm}$ sources, BIMA 1,2, and 3 from Beltrán et al. (2002), and the white one shows the position of the 1.3 mm continuum source C detected by Codella et al. (2001).

higher than that of Fig. 5 in Nisini et al. (2001). What is more, the interferometer has filtered out the extended emission of the outflow allowing closer study of the innermost part of the outflow and comparison of the correlation between the $\mathrm{H}_{2}$ and the $\mathrm{CO}$ emission. The bottom panels of Fig. 7 show a close-up image of the $\mathrm{H}_{2}$ emission towards the embedded YSOs BIMA 1, 2 , and 3, overlaid with the $\mathrm{CO}(J=1 \rightarrow 0)$ emission integrated in the intermediate- (Fig. 7b), and high-velocity outflow interval (Fig. 7c), and the CS ( $J=5 \rightarrow 4$ ) integrated emission (Fig. 7d) of the BIMA 2 outflow in red and blue contours (Beltrán et al. 2002).

Figure $7 \mathrm{c}$ shows that at high outflow velocities, the $\mathrm{CO}$ outflow with a well-defined bipolar structure stops before reaching the position of the $\mathrm{H}_{2}$ emission. Interestingly, on the west side, the $\mathrm{CO}$ emission stops in front of the strong $\mathrm{H}_{2}$ knots $\mathrm{K} 1$ and K2. The position of these two knots coincides with a blueshifted clump visible in CS $(J=5 \rightarrow 4)$ (Fig. 7d) and $\mathrm{CH}_{3} \mathrm{OH}(\mathrm{J}=5 \rightarrow 4)$ and identified as clump B by Beltrán et al. (2002). These authors suggest that there is a shocked surface at the position of this clump, which would be the responsible for the deflection and V-shaped morphology of the molecular outflow at low and intermediate outflow velocities (Figs. 7a,b) westwards of BIMA 2. The detection of the $\mathrm{H}_{2}$ knots $\mathrm{K} 1$ and $\mathrm{K} 2$ seems to confirm this scenario. Knot K3, the strand of knots I, and knot $\mathrm{H} 1$ are associated with the deflected $\mathrm{CO}$ emission (Figs. 7a,b). In particular, H1 is associated with a redshifted and blueshifted CO clump, also visible in CS $(J=2 \rightarrow 1)$ (Beltrán et al. 2002). Eastwards of BIMA 2, Beltrán et al. (2002) suggest that the redshifted clump visible in CS $(J=5 \rightarrow 4)$ (Fig. 7d) and $\mathrm{CH}_{3} \mathrm{OH}(\mathrm{J}=5 \rightarrow 4)$ and named $\mathrm{R}$ is responsible for the change in the velocity of the gas in the outflow. The eastern $\mathrm{CO}$ emission, between the driving source and clump $\mathrm{R}$, is mainly redshifted at intermediate and high outflow velocities, whereas farther away from clump $\mathrm{R}$ the outflow emission is slightly stronger in the blue wing than in the red wing. This clump $\mathrm{R}$, however, is not visible in $\mathrm{H}_{2}$ emission. A possible explanation for this could be that the $\mathrm{H}_{2}$ molecules are dissociated in the shock, and thus, there is not enough $\mathrm{H}_{2}$ to be detected. However, the most plausible explanation would be that the R clump, which is located towards the redshifted lobe of the outflow, is not visible in $\mathrm{H}_{2}$ due to the extinction produced by the circumstellar material surrounding BIMA 2. Submillimeter observations carried out with the James Clerk Maxwell Telescope by Correia (2000) have estimated a circumstellar mass of $\sim 20 M_{\odot}$ associated with BIMA 2 . There is indeed a small knot labeled S1 (Figs. 6d and 7d) on the red lobe side of the outflow. Although its position does not exactly coincide with that of the clump $\mathrm{R}$, we cannot discard the possibility that this knot is part of the shell of the deflecting clump, where the extinction is lower. The $\mathrm{H}_{2}$ emission is clearly detected farther east probably because the emission has shifted from redshifted to blueshifted and the $\mathrm{H}_{2}$ flow has reached outside the core surrounding BIMA 2. In fact, the $\mathrm{H}_{2}$ emission of 

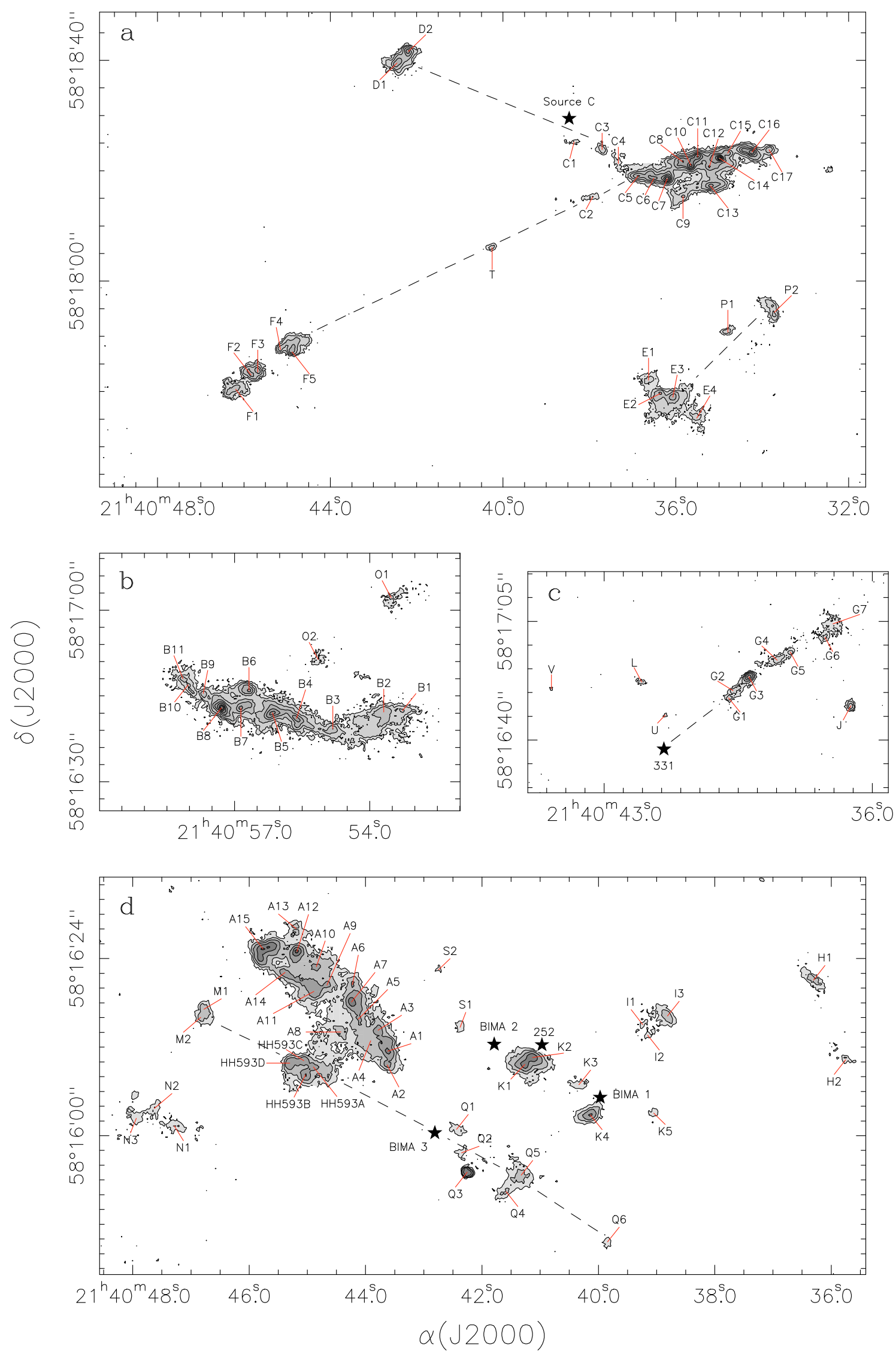

Fig. 6. $\mathrm{H}_{2}(2.12 \mu \mathrm{m})$ close-up images (continuum-subtracted) of the resolved knots in the jet features towards IC $1396 \mathrm{~N}$, including the names of all the knots. The contours are in units of $5.7 \times 10^{-16} \mathrm{erg} \mathrm{cm}^{-2} \mathrm{~s}^{-1} \operatorname{arcsec}^{-2}(1,2,4,8,13,20,28$, and 33). The black stars show the position of the $1.3 \mathrm{~mm}$ continuum source $\mathrm{C}$ detected by Codella et al. (2001) (top panel), of source \# 331 (middle right panel), and of source \# 252 and the $3.1 \mathrm{~mm}$ sources, BIMA 1, 2, and 3 detected by Beltrán et al. (2002) (bottom panel). The dashed lines indicate the orientation of the possible $\mathrm{H}_{2}$ flows described in the text (see Sect. 3.3). Due to the complexity of the flow powered by BIMA 2 (see Sect. 3.3.1), its direction is not indicated. 
the chain of knots A is clearly associated with the CO emission at low and intermediate outflow velocities (Figs. 7a,b). The knot labeled A15 (Fig. 6d), which is located at the tip of the chain of knots A, is probably a bow-shock, as suggested by its curved morphology and by its association with high-density gas as traced by CS $(J=5 \rightarrow 4)$ and $\operatorname{CS}(J=2 \rightarrow 1)$ (Fig. 7 and Beltrán et al. 2002).

The $\mathrm{CO}$ emission extends farther out towards the east, in the direction of the chain of knots B. Unfortunately, this feature is located too far from the phase center of the millimeter observations, so that the interferometer is not sensitive to the emission. In any case, Nisini et al. (2001) and Codella et al. (2001) show that there is also $\mathrm{CO}$ emission at the position of the strand of knots B. If the chain of knots A and B were both associated with the BIMA 2 outflow, then the length of this flow eastwards of BIMA 2 would be $\sim 2.3$ or $0.5 \mathrm{pc}$ at a distance of $750 \mathrm{pc}$, which corresponds to a dynamical timescale, $t_{\mathrm{dyn}}$, of $\sim 3300 \mathrm{yr}$ for a typical jet velocity, $v_{\text {jet }}$, of $150 \mathrm{~km} \mathrm{~s}^{-1}$ (Reipurth \& Bally 2001); however, to connect the intermediate-mass protostar BIMA 2 with the knots in $\mathrm{A}$ and the knots in $\mathrm{B}$, one cannot follow a straight line but a curved one (Fig. 7a). Such a curve is also visible in the $\mathrm{CO}$ emission and could suggest wiggling or precession of the flow. The precession of the BIMA 2 outflow could be possible if the powering source were a binary system instead. As a matter of fact, the intermediate-mass source BIMA 2 is not associated with a binary system but with a cluster, as recently reported by Neri et al. (2007), with at least 3 cores embedded inside a common envelope.

Regarding the other knots in the region, it is possible to trace a straight line to connect BIMA 3 with the group of knots labeled Q towards the southwest and with the knots HH593 and the knots M 1 and M 2 towards the northeast. This can be extrapolated up to reach the group of knots labeled B. In particular, the line can be traced towards knots B 1 and B 2 with a different orientation from the rest of knots in B. This, together with the bow-shock shape of knots Q4 and Q5 (Fig. 6d), leads us to speculate on the possibility that BIMA 3 is driving an additional flow, which could have a length from BIMA 3 to B 1 and B 2 of $\sim 1.6$ or $0.35 \mathrm{pc}$, and $t_{\text {dyn }} \simeq 2300 \mathrm{yr}$ for $v_{\text {jet }}=150 \mathrm{~km} \mathrm{~s}^{-1}$. The length of the flow from Q 6 to M 1 and M 2 is $\sim 1$ '. 1 or 0.24 pc. Although no $\mathrm{CO}(J=1 \rightarrow 0)$ emission has been detected towards the position of this possible outflow through interferometric observations (Fig. 7), Codella et al. (2001) have observed $\mathrm{CO}(J=2 \rightarrow 1)$ through single-dish observations; therefore, this could be an old and poor collimated outflow whose emission has been filtered out by the interferometer.

$\mathrm{No}_{2}$ emission has been clearly found in association with the north-south outflow mapped in $\mathrm{CO}(J=1 \rightarrow 0)$ by Beltrán et al. (2002) and proposed to be driven by BIMA 1, nor with a possible additional CS $(J=2 \rightarrow 1)$ outflow observed westwards of the BIMA 1 outflow (Beltrán et al. 2004).

\subsubsection{Other possible $\mathrm{H}_{2}$ flows in the globule}

One of the $\mathrm{H}_{2}$ features mapped in the region by Nisini et al. (2001) is the chain of knots labeled G. As seen in Fig. 6c, this strand of knots shows a jet-like morphology with an elongation of $\sim 0$.'75 or $\sim 0.16 \mathrm{pc}$. This possible flow seems to be emanating from source \# 331, named HH777 IRS by Reipurth et al. (2003), which is possibly binary as discussed in Sect. 3.1. One of the sources in this binary system could be driving the HH777 flow mapped by Reipurth et al. (2003). The HH777 flow has a PA of about $-120^{\circ}$. This flow is visible in $\mathrm{H}_{\alpha}$ and [SII] as a bright

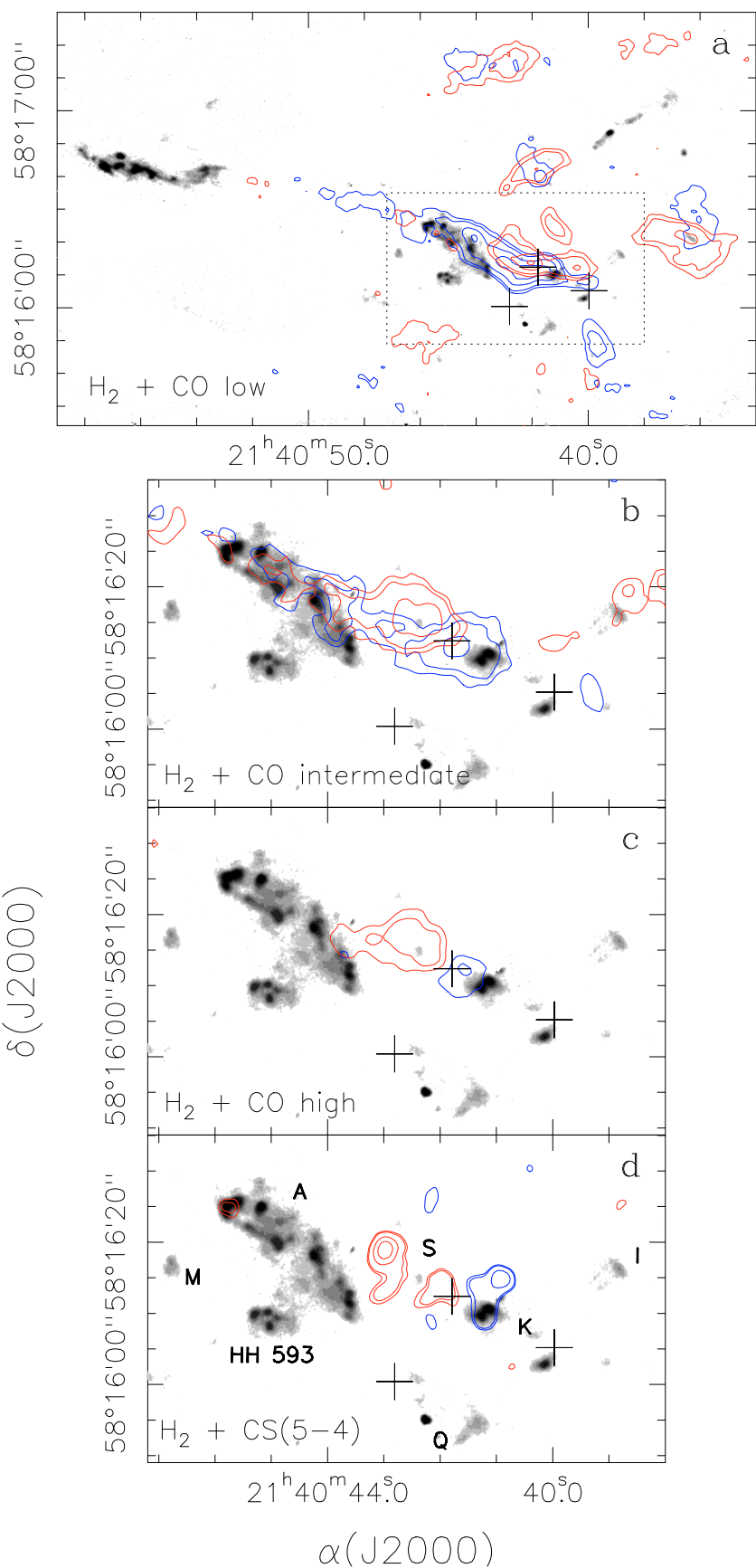

Fig. 7. a): $\mathrm{H}_{2}(2.12 \mu \mathrm{m})$ image (continuum-subtracted) in grey-scale and $\mathrm{CO}(J=1 \rightarrow 0)$ emission integrated in the $[ \pm 3.5, \pm 9.5] \mathrm{km} \mathrm{s}^{-1}$ velocity interval in red and blue contours (Beltrán et al. 2002). (Lower panels): $\mathrm{H}_{2}(2.12 \mu \mathrm{m})$ close-up image (continuum-subtracted), towards the position of BIMA 1,2 , and 3 , in grey-scale and CO $(J=1 \rightarrow 0)$ emission integrated in the the $[ \pm 9.5, \pm 15.5] \mathrm{km} \mathrm{s}^{-1}$ interval b), and the $[ \pm 15.5, \pm 21.5] \mathrm{km} \mathrm{s}^{-1}$ interval c), and CS $(J=5 \rightarrow 4)$ emission integrated in the $[ \pm 5, \pm 12] \mathrm{km} \mathrm{s}^{-1}$ velocity interval d), in red and blue contours (Beltrán et al. 2002). The black crosses show the positions of the $3.1 \mathrm{~mm}$ sources, BIMA 1, 2, and 3 from Beltrán et al. (2002).

working surface abruptly emerging from the southwestern sharp rim of the cloud core (Reipurth et al. 2003). The bow shock is also visible in the Digital Sky Survey 2 optical image (see Fig. 1 of Beltrán et al. 2002). Our $\mathrm{H}_{2}$ observations do not cover the position of this bow-shock. As for the $\mathrm{HH} 777$ flow before emerging from the rim, that is, closer to source \# 331, no $\mathrm{H}_{2}$ emission has been detected. Regarding the chain of knots $G$, it is not easily 
associated with any of the $\mathrm{CO}(J=2 \rightarrow 1)$ peaks mapped by Codella et al. (2001), although it is clearly located in a region of enhanced high-velocity $\mathrm{CO}$ emission (see Fig. 5 of Nisini et al. 2001). No CO $(J=1 \rightarrow 0)$ emission has been detected either in association with the $\mathrm{G}$ knots in the interferometric maps of Beltrán et al. (2002) (see Fig. 5). This could stem from this feature being located too far from the phase center of the millimeter observations that the interferometer is not sensitive to the emission. Small blueshifted and redshifted CO emission clumps have been detected towards the position of \# 331 (Fig. 5), and Caratti o Garatti et al. (2006) have detected [FeII] as well. The dynamical timescale of the possible $\mathrm{G}$ flow would be $\sim 1000 \mathrm{yr}$ for a typical $v_{\text {jet }}$. Interestingly, the strand of knots $\mathrm{G}$ points towards a northwestern cavity clearly visible in the IRAC 3.6, 4.5, 5.8, and $8.0 \mu \mathrm{m}$ images, which leads us to speculate on the possibility that such a cavity has been excavated by a molecular outflow that could be associated with these $\mathrm{H}_{2}$ knots.

Towards the north of the IC $1396 \mathrm{~N}$ globule there are some other prominent groups or chains of knots, labeled C, D, E, F, and P. Nisini et al. (2001) propose that the strands of knots E and $\mathrm{F}$ could be associated with a flow, which they call outflow II, and that the chains of knots $\mathrm{C}$ and D could be associated with the two lobes of a same flow, called outflow III. In the latter scenario, the powering source of the possible C-D flow would be the $1.3 \mathrm{~mm}$ continuum source $\mathrm{C}$ detected by Codella et al. (2001) and visible in the top panel of Fig. 6. However, as seen in Fig. 6a, the group of knots E seems to be more likely associated with those labeled $\mathrm{P}$, with the knot $\mathrm{P} 2$ showing a sort of bow-shock morphology. Regarding the flow $\mathrm{C}-\mathrm{D}$, there are some individual knots in the chain of knots $\mathrm{C}(\mathrm{C} 1, \mathrm{C} 3$, and $\mathrm{C} 4)$ expanding towards the group of knots $\mathrm{D}$, as one would expect in the scenario proposed by Nisini et al. (2001). The length of this flow would be $\sim 0$ '.84 or $\sim 0.18 \mathrm{pc}$. If the $1.3 \mathrm{~mm}$ continuum source $\mathrm{C}$ is powering the flow $\mathrm{C}-\mathrm{D}$, then its $t_{\text {dyn }}$ would be $\sim 750 \mathrm{yr}$ for a typical $v_{\text {jet }}$. It should be noted, however, that there are also some knots in $\mathrm{C}$ expanding towards the chain of knots $\mathrm{F}$, and there is an additional knot, labeled $\mathrm{T}$, located between the knots chains $\mathrm{F}$ and $\mathrm{C}$. This suggests a possible association of the strands of knots $\mathrm{C}, \mathrm{T}$, and $\mathrm{F}$ that could be part of the same flow (see Fig. 6a). The length of this possible flow would be $\sim 1.8$ or $\sim 0.40$ pc. In fact, Spizer $4.5 \mu \mathrm{m}$ observations seem to give support to this scenario, as the infrared emission connects F, T, and C (see Fig. 5). The two strands of knots C and $\mathrm{F}$ could be associated with the redshifted and blueshifted lobes, respectively, of the northern outflow orientated east-west mapped in $\mathrm{CO}(J=2 \rightarrow 1)$ by Codella et al. (2001). In this case, the redshifted lobe would be that of the outflow II, according to Nisini et al. (2001), while the blueshifted lobe would be that of the outflow III. The source powering this flow could be embedded in the dense gas detected in CS by Codella et al. (2001) and $\mathrm{H}^{13} \mathrm{CO}^{+}$by Sugitani et al. (2002a). An emission peak, labeled as Core I, is visible in the Sugitani et al. maps. The high-density gas emission coincides with a high extincted elongated region clearly visible in the $J$ and $H$ band maps (see Fig. 1). Therefore, a possible scenario could be the presence of two flows, one of them traced by the strands of knots F, T, and some knots of the $\mathrm{C}$ strand, and the other one by the group of knots D and some other individual knots of $\mathrm{C}$. These two flows would collide towards the position of the chain of knots $\mathrm{C}$, which would explain the strong $\mathrm{H}_{2}$ emission towards this feature. By extrapolating southwards the line that connects the chains of knots $\mathrm{C}, \mathrm{T}$, and F, one finds the group of knots labeled $\mathrm{O}$ and, by continuing farther south, the chain of knots B. Therefore, one could hypothesize that all these groups of knots could be related and be part of a long chain of $\mathrm{H}_{2}$ emission knots. The total length of this long $\mathrm{H}_{2}$ flow would be $\sim 3$ '. 1 or $\sim 0.70 \mathrm{pc}$ at the distance of IC $1396 \mathrm{~N}$.

From the morphology alone, it is difficult to confirm the possible flows observed in IC $1396 \mathrm{~N}$. To study the kinematics and physical conditions of the $\mathrm{H}_{2}$ emission and determine whether different $\mathrm{H}_{2}$ features that seem to be morphologically related are indeed part of the same long flow, additional long-slit NIR spectroscopy observations would be needed. In addition, if $v_{\text {jet }} \simeq 150 \mathrm{~km} \mathrm{~s}^{-1}$, then in about $\sim 5 \mathrm{yr}$ we should be able to crosscorrelate the images and measure displacements of the knots on the order of the pixel size of NICS at the TNG. This way one could determine proper motions that would help to confirm or discard possible flows.

\section{Summary and conclusions}

We carried out deep NIR observations at $J, H$, and $K^{\prime}$ with NICS at the TNG telescope to study the stellar population associated with the bright-rimmed cloud IC $1396 \mathrm{~N}$ in detail and reveal additional young sources inside the globule. The deep high angular resolution $\mathrm{H}_{2}$ observations helped us to investigate the complex structure of this globule and the morphology of the shocked gas that traces the interaction between the outflows in the region and the dense clumps surrounding the YSOs.

We found 736 sources detected in all three bands within the area where the $J H K^{\prime}$ images overlap $\left(\sim 4.2 \times 4.2 \operatorname{arcmin}^{2}\right)$. There are 128 sources detected only in $H K^{\prime}, 67$ detected only in $K^{\prime}$, and 79 detected only in $J H$. The completeness limits in the 2MASS standard are $K_{\mathrm{s}} \sim 17.5, H \sim 18.5$, and $J \sim 19.5$. The sources with only $H K^{\prime}$ or $K^{\prime}$ detections are primarily located towards the globule, whereas the sources with $J H$ detections tend to be located outside the globule. Most of the stars in the region either fall within the reddening band of the main sequence or exhibit only a small NIR excess as shown by the CCD. The source \# 331, which coincides with source \# 8 in Nisini et al. (2001) and HH777 IRS in Reipurth et al. (2003), is possibly a binary and the photometry has been derived for both A and B components. The sources of this system could be powering the major flow HH777 (Reipurth et al. (2003) and the $\mathrm{H}_{2}$ flow $\mathrm{G}$ (Nisini et al. 2001). Although there are signatures of star formation in the globule, such as molecular outflows and jets, only the source \# 331 A exhibits a large NIR excess. This and source \# 252 have been tentatively classified as Class I sources of intermediate mass based on the CMD.

We did not find any color or age gradient in the north-south direction, indicative of the triggered star formation scenario suggested by Getman et al. (2007) from X-ray observations. We also did not find any clear evidence of clustering of sources with NIR excess towards the southern edge of the globule. The evolutionary gradient found by Getman et al. (2007) may not correspond to an age gradient, since the intense UV radiation may have affected the circumstellar environments of the protostars close to the rim, suddenly stopping their growth and making them appear as less evolved Class II/III sources. What is clear from NIR and millimeter observations is that not all the star formation in the globule can be explained in terms of triggering.

The $\mathrm{H}_{2}$ emission is complex and knotty and shows several molecular hydrogen features spread over the region, testifying to recent star-formation activity throughout the whole globule. The $\mathrm{H}_{2}$ emission is resolved into several chains or groups of knots that sometimes show a jet-like morphology. This and the knots being located in different parts of the globule suggest that the $\mathrm{H}_{2}$ excitation mostly comes from shocks driven by outflows powered by YSOs. The shocked cloudlet model scenario proposed 
by Beltrán et al. (2002) to explain the V-shaped morphology of the CO molecular outflow powered by BIMA 2 seems to be confirmed by the presence of $\mathrm{H}_{2}$ emission (knots $\mathrm{K} 1$ and $\mathrm{K} 2$ ) at the position of the western clump $\mathrm{B}$, which is causing the deflection of the outflow. The eastern deflecting clump R, visible in highdensity tracers, is not visible in $\mathrm{H}_{2}$, but this could stem from extinction. The $\mathrm{H}_{2}$ emission of this BIMA 2 flow is visible farther east and is associated with the chain of knots A, probably when the emission reaches outside the core surrounding BIMA 2.

New possible flows have been discovered in the globule. One of them would be denoted by the group of knots $\mathrm{E}$ and those labeled P. Another flow would be traced by the strands of knots $\mathrm{F}$, T, and some knots of the $\mathrm{C}$ strand. Towards the position of the chain of knots $C$, this flow would collide with the previously known flow $\mathrm{C}-\mathrm{D}$, denoted by the group of knots $\mathrm{D}$ and some individuals knots of $\mathrm{C}$. The $\mathrm{C}-\mathrm{T}-\mathrm{F}$ flow could extend farther southwards up to reach the group of knots $\mathrm{O}$ or even the chain of knots B. Another possible flow has also been discovered towards the south of the globule that would be traced by the group of knots Q, HH593 and M, and could be powered by the YSO BIMA 3. This flow could extend up to reach some knots of the strand of knots B. To confirm these flows, additional long-slit NIR spectroscopy observations and proper motions determination would be needed.

Acknowledgements. This work is based on observations made with the Italian Telescopio Nazionale Galileo (TNG) operated on the island of La Palma by the Fundación Galileo Galilei of the INAF (Istituto Nazionale di Astrofisica) at the Spanish Observatorio del Roque de los Muchachos of the Instituto de Astrofisica de Canarias.

This work is based in part on observations made with the Spitzer Space Telescope, which is operated by the Jet Propulsion Laboratory, California Institute of Technology under a contract with NASA.

This publication makes use of data products from the Two Micron All Sky Survey, which is a joint project of the University of Massachusetts and the Infrared Processing and Analysis Center/California Institute of Technology, funded by the National Aeronautics and Space Administration and the National Science Foundation.

M.T.B., R.L., J.M.G., and R.E. are supported by MEC grant AYA200508523-C03. J.M.G. is also supported by AGAUR grant 2005SGR00489. F.M. acknowledges support from the Universitat de Barcelona during the data calibration process. This publication makes use of data products from the Two Micron All Sky Survey, which is a joint project of the University of Massachusetts and the Infrared Processing and Analysis Center/California Institute of Technology, funded by the National Aeronautics and Space Administration and the National Science Foundation.

\section{References}

Allen, C. W. 1976, Astrophysical Quantities 3rd edn (London: Athlone press) Baffa, C., Comoretto, G., Gennari, S., et al. 2001, A\&A, 378, 722

Beltrán, M. T., Girart, J. M., Estalella, R., Ho, P. T. P., \& Palau, A. 2002, ApJ, 573,246

Beltrán, M. T., Girart, J. M., Estalella, R., \& Ho, P. T. P. 2004, A\&A, 426, 941

Caratti o Garatti, A., Giannini, T., Nisini, B., \& Lorenzetti, D. 2006, A\&A, 449, 1077

Carpenter, J. M. 2001, AJ, 121, 285

Codella, C., Bachiller, R., Nisini, B., Saraceno, P., \& Testi, L. 2001, A\&A, 376, 271

Correia, J. C. 2000, Ph.D. Thesis, Univ. London

Getman, K. V., Feigelson, E. D., Garmire, G., Broos, P., \& Wang, J. 2007, ApJ, 654,316

Koornneef, J. 1983, A\&A, 128, 84

Lada, C. J., \& Lada, E. A. 2003, ARA\&A, 41, 57

Matthews, T. J. 1979, A\&A, 75, 345

Neri, R., Fuente, A., Ceccarelli, C., Caselli, P., et al. 2007, A\&A, 468, L33

Nisini, B., Massi, F., Vitali, F., Giannini, T., et al. 2001, A\&A, 376, 553

Palla, F., \& Stahler, S. W. 1991, ApJ, 375, 288

Palla, F., \& Stahler, S. W. 1999, ApJ, 525, 772

Reipurth, B., \& Bally, J. 2001, ARA\&A, 39, 403

Reipurth, B., Armond, T., Raga, A., \& Bally, J. 2003, ApJ, 593, L47

Rieke, G. H., \& Lebofsky, M. J. 1985, ApJ, 288, 618

Saraceno, P., Ceccarelli, C., Clegg, P., Correia, C., et al. 1996, A\&A, 315, L293

Smith, M. D., \& Rosen, A. 2005, MNRAS, 357, 1370

Sugitani, K., Fukui, Y., Mizuno, A., \& Ohashi, N. 1989, ApJ, 342, L87

Sugitani, K., Fukui, Y., \& Ogura, K. 1991, ApJS, 77, 59

Sugitani, K., Tamura, M., \& Ogura, K. 1995, ApJ, 455, L39

Sugitani, K., Tamura, M., Nakaya, H., Nakajima, Y., et al. 2002a, Proceedings of the IAU 8th Asian-Pacific Regional Meeting, Vol. II, ed. J. Ikeuchi, Hearnshaw, \& T. Hanawa, (Tokyo: ASJ), 213

Sugitani, K., Tamura, M., Nakajima, Y., Nagashima, C., et al. 2002b, ApJ, 565, L25

Vacca, W. D., Garmany, C. D., \& Shull, J. M. 1996, ApJ, 460, 914

Vanbeveren, D., De Loore, C., \& Van Rensbergen, W. 1998, A\&AR, 9, 63

Walborn, N. R., \& Panek, R. J. 1984, ApJ, 286, 718 